\title{
Statistical inference for generalized Pareto distribution based on progressive Type-II censored data with random removals
}

\author{
Reza Azimi *, Bahman Fasihi, Faramarz Azimi Sarikhanbaglu \\ Department of Statistics, Parsabad Moghan Branch, Islamic Azad University, Parsabad Moghan, Iran \\ *Corresponding author E-mail: azimireza1365@gmail.com
}

Copyright (C)2014 Reza Azimi et. al. This is an open access article distributed under the Creative Commons Attribution License, which permits unrestricted use, distribution, and reproduction in any medium, provided the original work is properly cited.

\begin{abstract}
In this article, We consider the estimation problems of the parameter and reliability function of the generalized Pareto distribution based on a progressively type-II censored sample with random (Binomial) removals. we use the method of maximum likelihood and Bayesian estimation to estimate parameter and reliability function. Bayesian estimates are derived under squared error and LINEX loss functions. we also construct the confidence interval for the parameter of generalized Pareto distribution based on a progressively type-II censored sample with random removals. The comparisons between different estimators are made based on simulation study.
\end{abstract}

Keywords: Generalized Pareto distribution, progressive Type-II censored, random removals, Bayesian estimates, reliability function

\section{Introduction}

In various life-testing and reliability studies, experiments must often terminate before all units on test have failed. In such cases, one has complete information only on part of the sample. On all units which have not failed, one has only partial information. Such data are called censored. There are several types of censored tests. One of the most common censored tests is progressive type II censoring. In progressive type II censoring, Suppose that $n$ units are placed on a life test and the experimenter decides beforehand quantity $m$, the number of units, to be failed. Now at the time of the first failure, $R_{1}$ of the remaining $n-1$ surviving units are randomly removed from the experiment. Continuing on, at the time of the second failure, $R_{2}$ of the remaining $n-R_{1}-2$ units are randomly drawn from the experiment. Finally, at the time of the $m$ th failure, all the remaining $R_{m}=n-m-R_{1}-R_{2}-\ldots-R_{m-1}$ surviving units are removed from the experiment. Note that, in this scheme, $R_{1}, R_{2}, \ldots, R_{m}$ are all pre-fixed. However, in some practical situations, these numbers may occur at random. for example, in some reliability experiments, an experimenter may decide that it is inappropriate or too dangerous to carry on the testing on some of the tested units even though these units have not failed. In such cases, the pattern of removal at each failure is random (Zeinab [3], Yen and Tse [2]). This leads to progressive censoring with random removals (illustrated by "Table 1").

There have been several references about the statistical inference on lifetime distributions under progressive censoring with random removals; for example, we refer to Yuen and Tse [2], Tse and Yuen [5], Tse et al. [10], Shuo and Tao [12], Wu [11], Wu and Chang [4] and $\mathrm{Wu}$ et al. [9].

Based on a progressively type-II censored sample with random removals, we consider the problem of estimating 
Table 1: A schematic representation of the progressive type-II censoring with binomial removals

\begin{tabular}{ccccc}
\hline Process & $\begin{array}{c}\text { The number } \\
\text { in life testing }\end{array}$ & Failures & Binomial Removals & Remains \\
\hline 1 & $n$ & 1 & $R_{1} \sim B(n-m, p)$ & $n-1-R_{1}$ \\
2 & $n-1-R_{1}$ & 1 & $R_{2} \sim B\left(n-m-R_{1}, p\right)$ & $n-2-R_{1}-R_{2}$ \\
$\ldots$ & $\ldots$ & $\ldots$ & $\ldots$ & $\cdots$ \\
$m-1$ & $n-(m-2)-\sum_{j=1}^{m-2} R_{j}$ & 1 & $R_{m-1} \sim B\left(n-m-\sum_{j=1}^{m-2} R_{j}, p\right)$ & $n-(m-1)-\sum_{j=1}^{m-1} R_{j}$ \\
$m$ & $n-(m-1)-\sum_{j=1}^{m-1} R_{j}$ & 1 & $R_{m}=n-m-\sum_{j=1}^{m-1} R_{j}$ & 0 \\
\hline
\end{tabular}

parameter and reliability function of the two parameter generalized Pareto distribution with the shape parameter $\theta$ and the scale parameter $\sigma$, proposed by Castillo and Hadi [7]. under both classical and Bayesian (with different loss functions) contexts.

The cumulative distribution function, probability density and reliability functions of two parameter generalized Pareto distribution are respectively given by

$F(x \mid \sigma, \theta)=1-\left(1-\frac{x}{\sigma}\right)^{\frac{1}{\theta}} ; \theta>0,0<x<\sigma$

and

$f(x \mid \sigma, \theta)=\frac{1}{\theta \sigma}\left(1-\frac{x}{\sigma}\right)^{\frac{1}{\theta}-1} ; \theta>0,0<x<\sigma$

and

$R(x \mid \sigma, \theta)=\left(1-\frac{x}{\sigma}\right)^{\frac{1}{\theta}} ; \theta>0,0<x<\sigma$

for more detail about two parameter generalized Pareto distribution see Castillo and Hadi [7]. two parameter generalized Pareto distribution were widely used by several authors, Among others, we refer to Grimshaw [6], Castillo and Hadi [7], and Castillo et al. [8].

\section{Maximum likelihood estimation}

Let $X_{1}<X_{2}<\ldots<X_{m}$ be the ordered failure times out of $n$ randomly selected times, where $m$ is predetermined before the test. At the $i$ th failure, $R_{i}$ items are removed from the test. For progressive censoring with pre-determined number of removals $R=\left(R_{1}=r_{i}, \ldots, R_{m-1}\right)=r_{m-1}$, the likelihood function can be defined as the following form

$L(\sigma, \theta, x \mid R)=c \prod_{i=1}^{m} f\left(x_{i} \mid \sigma, \theta\right)\left[1-F\left(x_{i} \mid \sigma, \theta\right)\right]^{r_{i}}$

where $c=n\left(n-1-R_{1}\right) \cdots\left(n-\sum_{i=1}^{m-1}\left(R_{i}+1\right)\right)$. Equation (3) is derived conditional on $R_{i}$. Each $R_{i}$ can be of any integer value between 0 and $n-m-\sum_{j=1}^{i-1}\left(R_{j}\right)$. It is different from progressive censoring with fixed removal that $R_{i}$ is a random number and is assumed to follow a binomial distribution with parameter $p$. It means that each unit leaves with equal probability $p$ and the probability of $R_{i}$ units leaving after the $i$ th failure occurs is

$P\left(R_{1}=r_{1}\right)=p^{r_{1}}(1-p)^{n-m-r_{1}}$

$P\left(R_{i}=r_{i} \mid R_{i-1}=r_{i-1}, \ldots, R_{1}=r_{1}\right)=\left(n-m-\sum_{j=1}^{i-1} r_{j}\right) p^{r_{i}}(1-p)^{n-m-\sum_{j=1}^{m-1} r_{j}}$ 
where $0 \leq r_{i} \leq n-m-\sum_{j=1}^{i-1} r_{j}(i=1, \ldots, m-1)$. Furthermore, we assume that $R_{i}$ is independent of $X_{i}$ for all $i$. The joint likelihood function of $X=\left(X_{1}, X_{2}, \ldots, X_{m}\right)$ and $R=\left(r_{1}, r_{2}, \ldots, r_{m}\right)$ can be found as

$L(\sigma, \theta, x, p)=L(\sigma, \theta, x \mid R) P(R, p)$

where $P(R, p)$ is the joint probability distribution of $R=\left(r_{1}, r_{2}, \ldots, r_{m}\right)$ and in particular

$$
\begin{array}{r}
P(R, p)=P\left(R_{m}=r_{m} \mid R_{m-1}=r_{m-1}, \ldots, R_{1}=r_{1}\right) \times \ldots \\
\times P\left(R_{2}=r_{2} \mid R_{1}=r_{1}\right) P\left(R_{1}=r_{1}\right)
\end{array}
$$

Therefore

$$
P(R, p)=\frac{(n-m) !}{\left(n-m-\sum_{j=1}^{m-1} r_{j}\right) ! \prod_{j=1}^{m-1} r_{j}} p^{\sum_{j=1}^{m-1} r_{j}}(1-p)^{(m-1)(n-m)-\sum_{j=1}^{m-1}(m-j) r_{j}}
$$

Substituting (1) and (2) into (4), the likelihood function takes the following form

$$
\begin{aligned}
L(\sigma, \theta, x, p) & \propto \sigma^{-m} \theta^{-m} \exp \left\{\frac{1}{\theta} \sum_{i=1}^{m}\left(r_{i}+1\right) \log \left(1-\frac{x_{i}}{\sigma}\right)\right\} \\
& \times \exp \left\{-\sum_{i=1}^{m} \log \left(1-\frac{x_{i}}{\sigma}\right)\right\} p^{\sum_{j=1}^{m-1} r_{j}}(1-p)^{(m-1)(n-m)-\sum_{j=1}^{m-1}(m-j) r_{j}} I_{(0, \sigma)}\left(x_{i}\right)
\end{aligned}
$$

The first partial derivatives of log-likelihood function with respect to $\theta$ is

$$
\frac{\partial \log L(\sigma, \theta, x, p)}{\partial \theta}=-\frac{m}{\theta}-\frac{\sum_{i=1}^{m}\left(r_{i}+1\right) \log \left(1-\frac{x_{i}}{\sigma}\right)}{\theta^{2}}=0
$$

therefor we get the MLE of $\theta$ as in the following form

$\hat{\theta}=-\frac{\sum_{i=1}^{m}\left(r_{i}+1\right) \log \left(1-\frac{x_{i}}{\sigma}\right)}{m}$ by

By the invariant property of the MLE, the MLE of the reliability function, $S=R(t)$, with fixed $t>0$. is given

$$
\hat{S}_{M L E}=\left(1-\frac{t}{\sigma}\right)^{\frac{1}{\hat{\theta}_{M L E}}}
$$

\section{Exact interval estimation}

Let $X_{1}<X_{2}<\cdots<X_{m}$ be a progressively type II censored sample from the generalized Pareto distribution . Furthermore, let $Y_{i}=-\frac{1}{\theta} \ln \left(1-\frac{x_{i}}{\sigma}\right), i=1, \ldots, m$. It is easy to show that $Y_{1}, \cdots, Y_{m}$ is a progressively Type II censored sample from the standard exponential distribution. For a fixed set of $R=\left(r_{1}, \ldots, r_{m}\right)$, let us consider the following transformation:

$Z_{1}=n Y_{1}$

$Z_{2}=\left(n-r_{1}-1\right)\left(Y_{2}-Y_{1}\right)$ 
$Z_{m}=\left(n-r_{1}-\cdots-r_{m}-m+1\right)\left(Y_{m}-Y_{m-1}\right)$

Balakrishnan and Aggarwala [1] showed that the progressively type II right censored spacings $Z_{1}, Z_{2}, \ldots, Z_{m}$ as defined in equation (8), are independent and identically distributed as a standard exponential distribution. Hence, $2 Z_{1}$ has a chi-square distribution with 2 degrees of freedom. Now, let

$W=2 \sum_{i=1}^{m} Z_{i}=-\frac{2}{\theta} \sum_{i=1}^{m}\left(r_{i}+1\right) \log \left(1-\frac{x_{i}}{\sigma}\right)=\frac{2 m \hat{\theta}}{\theta}$

It is easy to see that $W$ has a $\chi^{2}$ distribution with $2 m$ degrees of freedom. Confidence interval for $\theta$ can be obtained through the pivotal quantity $\frac{2 m \hat{\theta}}{\theta} \sim \chi_{(2 m)}^{2}$. Since the pivotal quantity $\frac{2 m \hat{\theta}}{\theta} \sim \chi_{(2 m)}^{2}$, then we have

$$
\begin{aligned}
1-\alpha= & P\left(\chi_{1-\frac{\alpha}{2}}^{2}(2 m)<\frac{2 m \hat{\theta}}{\theta}<\chi_{\frac{\alpha}{2}}^{2}(2 m)\right) \\
& =P\left(\frac{2 m \hat{\theta}}{\chi_{1-\frac{\alpha}{2}}^{2}(2 m)}<\theta<\frac{2 m \hat{\theta}}{\chi_{\frac{\alpha}{2}}^{2}(2 m)}\right) \\
& =P\left(\frac{-2 \sum_{i=1}^{m}\left(r_{i}+1\right) \log \left(1-\frac{x_{i}}{\sigma}\right)}{\chi_{1-\frac{\alpha}{2}}^{2}(2 m)}<\theta<\frac{-2 \sum_{i=1}^{m}\left(r_{i}+1\right) \log \left(1-\frac{x_{i}}{\sigma}\right)}{\chi_{\frac{\alpha}{2}}^{2}(2 m)}\right)
\end{aligned}
$$

\section{Bayesian estimation}

In the following, we present Bayes estimators of the shape parameter and reliability function of generalized Pareto distribution when samples are drawn from progressively Type-II censoring data with binomial removals For this purpose we assume the parameters $\theta$ and $p$ behave as independent random variables. In this paper, for parameter $\theta$ we consider inverted-gamma prior distribution of the form

$\pi_{1}(\theta)=\frac{\beta^{\alpha}}{\Gamma(\alpha)} \theta^{-(\alpha+1)} e^{-\left(\frac{\beta}{\theta}\right)} ;(\alpha, \beta)>0$

Independently from parameter $\theta, p$ has a beta prior distribution with parameters $a$ and $b$ of the form

$\pi_{2}(p)=\frac{1}{B(a, b)} p^{a-1}(1-p)^{b-1}, 0<p<1 ; \quad(a, b)>0$

Based on the prior $\pi_{1}(\theta)$ and $\pi_{2}(p)$, the joint prior $\operatorname{PDF}$ of $(\theta, p)$ is

$\pi(\theta, p)=\pi_{1}(\theta) \pi_{2}(p), \theta>0, \quad 0<p<1$

$$
=\frac{\beta^{\alpha}}{\Gamma(\alpha) B(a, b)} p^{a-1}(1-p)^{b-1} \theta^{-(\alpha+1)} e^{-\left(\frac{\beta}{\theta}\right)} ; \theta>0, \quad 0<p<1
$$

It follows, from (3) and(10), that the joint posterior distribution of $(\theta, p)$ is

$\pi(\theta, p \mid \mathbf{x}, \mathbf{r})=\frac{\beta^{* \alpha^{*}}}{\Gamma\left(\alpha^{*}\right) B\left(a^{*}, b^{*}\right)} \theta^{-\left(\alpha^{*}+1\right)} e^{-\frac{\beta^{*}}{\theta}} p^{a^{*}-1}(1-p)^{b^{*}-1}$

Where $\alpha^{*}=m+\alpha, \beta^{*}=\left(\beta-\sum_{i=1}^{m}\left(r_{i}+1\right) \log \left(1-\frac{x_{i}}{\sigma}\right)\right), a^{*}=a+\sum_{j=1}^{m-1} r_{j} b^{*}=b+(m-1)(n-m) \sum_{j=1}^{m-1}(m-j) r_{j}$ Therefore, the marginal posterior PDFs of $\theta$ and $p$ are given respectively by

$\pi(\theta \mid \mathbf{x}, \mathbf{r})=\frac{\beta^{* \alpha^{*}}}{\Gamma\left(\alpha^{*}\right)} \theta^{-\left(\alpha^{*}+1\right)} e^{-\frac{\beta^{*}}{\theta}}$

and

$\pi(p \mid \mathbf{x}, \mathbf{r})=\frac{1}{B\left(a^{*}, b^{*}\right)} p^{a^{*}-1}(1-p)^{b^{*}-1}$ 


\subsection{Bayesian Estimation Under squared error loss function}

Under SE loss function (symmetric), the estimator of a parameters is the posterior mean. Thus, Bayes estimators of the parameter $\theta$ is obtained by using the posterior density (12)

$\hat{\theta}_{S}=E(\theta \mid \mathbf{x}, \mathbf{r})=\frac{\beta-\sum_{i=1}^{m}\left(r_{i}+1\right) \log \left(1-\frac{x_{i}}{\sigma}\right)}{m+\alpha-1}$

\subsection{Bayesian Estimation under LINEX loss function}

The LINEX loss function for $\theta$ can be expressed as the following proportional(see Basu and Ebrahimi [13])

$L(\Delta) \propto \exp (c \Delta)-c \Delta-1 ; \quad c \neq 0$

where $\Delta=\frac{\hat{\theta}-\theta}{\theta}$ and $\hat{\theta}$ is an estimate of $\theta$. The Bayes estimator of $\theta$, denoted by $\hat{\theta_{L}}$ under the LINEX loss function is the solution of the following equation.

$E\left[\frac{1}{\theta} \exp \left(\frac{c \hat{\theta}_{L}}{\theta}\right) \mid \mathbf{x}, \mathbf{r}\right]=e^{c} E\left[\frac{1}{\theta} \mid \mathbf{x}, \mathbf{r}\right]$

Therefore we have

$\hat{\theta}_{L}=\frac{\beta-\sum_{i=1}^{m}\left(r_{i}+1\right) \log \left(1-\frac{x_{i}}{\sigma}\right)}{c}\left(1-e^{-\frac{c}{\alpha+m+1}}\right)$

\section{Bayesian estimation of reliability function $S=R(t)$}

Other problems of interest are those of estimating the reliability function $R(t)$, with fixed $t>0$. Let the reliability $S=R(t)$ be a parameter itself. replacing $\theta=\frac{\ln \frac{\sigma}{\sigma-t}}{-\ln S}$ in terms of $S$ by that of equation (12), we obtain the posterior density function $S$ as

$\pi(S \mid \mathbf{X})=\frac{\nu\left(x_{i}, t\right)^{\alpha^{*}}}{\Gamma\left(\alpha^{*}\right)}(-\ln s)^{\alpha^{*}-1} s^{\nu\left(x_{i}, t\right)-1}$

where,

$\nu\left(x_{i}, t\right)=\frac{\beta^{*}}{\ln \frac{\sigma}{\sigma-t}}$

By using posterior density function $S(15)$, the Bayes estimate of the $S=R(t)$ relative to quadratic loss is

$\hat{S}_{S}=\left(\frac{\nu\left(x_{i}, t\right)}{\nu\left(x_{i}, t\right)+1}\right)^{\alpha^{*}}$

Under LINEX loss function, the Bayes estimate of $S=R(t)$ using equation (15) is

$\hat{S}_{L}=-\frac{1}{c} \ln \left[\sum_{l=0}^{\infty} \frac{(-c)^{l}}{l !}\left(\frac{\nu\left(x_{i}, t\right)}{\nu\left(x_{i}, t\right)+l}\right)^{\alpha^{*}}\right]$

\section{Numerical study}

In this section, a Monte Carlo simulation study is conducted with various choices of sample sizes. We firstly generate the numbers of progressive censoring with binomial removals $r_{i}(i=1,2, \ldots, m)$, and progressive censoring with binomial removals samples generated from generalized Pareto distribution by using the algorithm described in Balakrishnan and Aggarwala [1]. We used the following steps to generate a progressive censoring with binomial removals samples generated from generalized Pareto distribution 
1. Generate a group values

$$
\begin{aligned}
& r_{i} \sim \operatorname{Binomial}\left(n-m-\sum_{j=1}^{i-1} r_{j}, p\right), \\
& r_{m}=n-m-\sum_{i=1}^{m-1} r_{i} \quad, i=1,2, \ldots, m-1
\end{aligned}
$$

according to the relevant value of $p$.

2. Simulate $m$ independent exponential random variables $Z_{1}, Z_{2}, \ldots, Z_{m}$.

This can be done using inverse transformation $Z_{i}=-\ln \left(1-U_{i}\right)$ where $U_{i}$ are independent uniform $(0,1)$ random variables.

3. Set

$$
X_{i}=\frac{Z_{1}}{n}+\frac{Z_{2}}{n-R_{1}-1}+\frac{Z_{3}}{n-R_{1}-R_{2}-2}+\cdots+\frac{Z_{i}}{n-R_{1}-R_{2}-\cdots-R_{i-1}-i+1}
$$

for $i=1,2, \ldots, m$. This is the required progressively type-II censored sample with binomial removals from the standard exponential distribution.

4. Finally, we set $Y_{i}=F^{-1}\left(1-\exp \left(-X_{i}\right)\right)$, for $i=1,2, \ldots, m$, where $F^{-1}($.$) is the inverse cumulative distribution$ function of the generalized Pareto distribution. Then $Y_{1}, Y_{2}, \cdots, Y_{m}$ is the required progressively type-II censored sample with binomial removals from the generalized Pareto distribution.

5. We compute the MLE of $\theta$ and $R(t)=S$ by (6) and (7).

6. We compute the the Bayes estimates $\theta$ and $R(t)=S$ by respectively, using (13), (14), (16) and (17).

7. We compute the confidence interval of $\theta$ by using (9).

8. We repeat the above steps 2000 times. We then obtain the means and the MSEs (mean squared error), where

$$
M S E=2000^{-1} \sum_{i=1}^{2000}(\phi-\hat{\phi})^{2}
$$

and $\hat{\phi}$ is the estimator of $\phi$

In all above cases the prior parameters chosen as $(\alpha=2, \beta=1)$ which yield the generated value of $\theta=2$ as the true value. The true values of $R(t)$ in $t=0.5$ is obtained $R(0.5)=0.9486833$. The results are summarized in Tables 2-5.

\section{Conclusion}

This paper presents different methods of estimation to estimate parameter and reliability function of two parameter generalized Pareto distribution based on a progressively type-II censored sample with random removals. Our observations about the results are stated in the follow:

- Table 2 and 4 shows that the Bayes estimates under squared error loss function has the smallest MSE's as compared with other estimates (Bayes estimates under the LINEX loss function and maximum likelihood estimator. However, maximum likelihood estimator method relatively more accurate estimators as compared with the Bayes estimation. It is immediate to note that the MSE's decrease as sample size $n$ increases.

- Table 3 and 5 shows that the Bayes estimates of reliability function under squared error loss function has the smallest estimated MSE's as compared with Bayes estimates under the LINEX loss function and maximum likelihood estimator. However, Bayes estimates under the LINEX loss function relatively more accurate estimators as compared with the maximum likelihood estimator and Bayes estimates under squared error loss function. Also, the MSE's decrease as $n$ increases.

- Table 2 and 4 shows that for different size $n$ and $m$, the width of the Confidence interval for $\theta$, decrease as $n$ and $m$ increases. 
Table 2: $\quad$ Averaged values of MSEs for estimates of $\theta,(P=0.1)$

\begin{tabular}{cc|c|ccccc}
\hline$n$ & $m$ & Generated $R_{i}$ & $\hat{\theta}_{M L E}$ & $\hat{\theta}_{S E}$ & $\hat{\theta}_{L I}(c=0.1)$ & $\hat{\theta}_{L I}(c=-0.1)$ & $\% 95 C I$ \\
& & & MSE & MSE & MSE & MSE & Width \\
\hline 10 & 5 & $(1,1,0,0,3)$ & 2.0097 & 1.8414 & 1.3724 & 1.3897 & $(0.9811,6.1896)$ \\
& & & 0.7815 & 0.56781 & 0.6952 & 0.6814 & 5.2089 \\
\cline { 3 - 8 } & 7 & $(0 * 5,2,1)$ & 2.0138 & 1.8847 & 1.5002 & 1.5153 & $(1.0779,5.0021)$ \\
& & & 0.5887 & 0.4639 & 0.5352 & 0.5261 & \\
\hline 20 & \multirow{2}{*}{5} & $\left(0,1,2,2,0^{*} 5,5\right)$ & 2.0144 & 1.9222 & 1.6202 & 1.6327 & $(1.1790,4.2008)$ \\
& & & 0.4066 & 0.3419 & 0.3828 & 0.3772 & \\
& \multirow{2}{*}{15} & $\left(0,1,0^{*} 8,1,0,1,0,2\right)$ & 1.9976 & 1.9353 & 1.7155 & 1.7250 & $(1.2756,3.5692)$ \\
& & & 0.2795 & 0.2499 & 0.2740 & 0.2708 & \\
\hline 30 & 20 & $\left(4,0,1,0^{*} 7\right.$ & 2.0150 & 1.9667 & 1.7918 & 1.7996 & $(1.3582,3.2989)$ \\
& & $\left., 1,0,1,0^{*} 5,1,2\right)$ & 0.2007 & 0.1829 & 0.1942 & 0.1924 & \\
\cline { 3 - 8 } & 25 & $\left(0^{*} 3,1,2,0,0\right.$ & 2.0226 & 1.9832 & 1.8383 & 1.8449 & $(1.4159,3.1254)$ \\
& & $\left., 1,0^{*} 16,1\right)$ & 0.1670 & 0.1542 & 0.1584 & 0.1572 & \\
\hline 40 & 30 & $\left(1,2,0^{*} 5,1,0,2,0,1\right.$ & 2.0131 & 1.9805 & 1.8576 & 1.8632 & $(1.4501,2.9838)$ \\
& & $\left., 0^{*} 4,1,0^{*} 4,1,0^{*} 7,1\right)$ & 0.1285 & 0.1206 & 0.1260 & 0.1251 & \\
& \multirow{2}{*}{35} & $(0,0,1,0,1,0$, & 2.0063 & 1.9783 & 1.8717 & 1.8767 & $(1.4779,2.8804)$ \\
& & $\left.1,0,0,2,0^{*} 25\right)$ & 0.1132 & 0.1074 & 0.1122 & 0.1114 & \\
\hline
\end{tabular}

Table 3: Averaged values of MSEs for estimates of the reliability function, $P=0.1$

\begin{tabular}{cc|cccc}
\hline$n$ & $m$ & $\hat{S}_{M L E}$ & $\hat{S}_{S}$ & $\hat{S}_{L}(c=0.1)$ & $\hat{S}_{L} c=-0.1$ \\
& & $\mathrm{MSE}$ & $\mathrm{MSE}$ & $\mathrm{MSE}$ & $\mathrm{MSE}$ \\
\hline 10 & 5 & 0.92483 & 0.93723 & 0.92896 & 0.92868 \\
& & 0.001604 & 0.001219 & 0.001454 & 0.001457 \\
\cline { 3 - 6 } & 7 & 0.93153 & 0.94101 & 0.93576 & 0.93543 \\
& & 0.000951 & 0.000702 & 0.000842 & 0.000847 \\
\hline 20 & 10 & 0.93676 & 0.94367 & 0.94107 & 0.94071 \\
& & 0.000544 & 0.000401 & 0.000471 & 0.000474 \\
\cline { 2 - 6 } & \multirow{2}{*}{15} & 0.94029 & 0.94505 & 0.94466 & 0.94428 \\
& & 0.000305 & 0.000231 & 0.000258 & 0.000260 \\
\hline 30 & 20 & 0.94298 & 0.94656 & 0.94738 & 0.94700 \\
& & 0.000188 & 0.000151 & 0.000162 & 0.000162 \\
\cline { 2 - 6 } & 25 & 0.94433 & 0.94720 & 0.94875 & 0.94836 \\
& & 0.000140 & 0.000117 & 0.000125 & 0.000125 \\
\hline 40 & 30 & 0.94502 & 0.94743 & 0.94945 & 0.94906 \\
& & $1.056 \mathrm{e}-04$ & $8.948 \mathrm{e}-05$ & $9.549 \mathrm{e}-05$ & $9.466 \mathrm{e}-05$ \\
\hline & 35 & 0.94537 & 0.94744 & 0.94981 & 0.94941 \\
& & $9.127 \mathrm{e}-05$ & $7.855 \mathrm{e}-05$ & $8.391 \mathrm{e}-05$ & $8.286 \mathrm{e}-05$ \\
\hline
\end{tabular}


Table 4: Averaged values of MSEs for estimates of $\theta,(P=0.6)$

\begin{tabular}{|c|c|c|c|c|c|c|c|}
\hline$n$ & $m$ & Generated $R_{i}$ & $\begin{array}{l}\hat{\theta}_{M L E} \\
\text { MSE }\end{array}$ & $\begin{array}{l}\hat{\theta}_{S E} \\
\text { MSE }\end{array}$ & $\begin{array}{c}\hat{\theta}_{L I}(c=0.1) \\
\operatorname{MSE}\end{array}$ & $\begin{array}{c}\hat{\theta}_{L I}(c=-0.1) \\
\operatorname{MSE}\end{array}$ & $\begin{array}{l}\text { \%95CI } \\
\text { Width }\end{array}$ \\
\hline \multirow[t]{4}{*}{10} & 5 & $(4,0,1,0,0)$ & 2.0142 & 1.8452 & 1.3753 & 1.3926 & $(0.9833,6.2035)$ \\
\hline & & & 0.8381 & 0.6058 & 0.7135 & 0.7003 & \\
\hline & 7 & $(3,0 * 6)$ & 2.0329 & 1.9038 & 1.5154 & 1.5307 & $(1.0896,5.0564)$ \\
\hline & & & 0.5661 & 0.4419 & 0.5089 & 0.49991 & \\
\hline \multirow[t]{4}{*}{20} & 10 & $(7,2,1,0 * 7)$ & 2.0113 & 1.9194 & 1.6179 & 1.6304 & $(1.1773,4.1944)$ \\
\hline & & & 0.3999 & 0.3369 & 0.3807 & 0.3750 & \\
\hline & 15 & $(2,1,2,0 * 12)$ & 1.9959 & 1.9337 & 1.7141 & 1.7236 & $(1.2745,3.5662)$ \\
\hline & & & 0.2752 & 0.2463 & 0.2718 & 0.2685 & \\
\hline \multirow[t]{4}{*}{30} & 20 & $(3,3,1,0,1,2,0 * 14)$ & 1.9946 & 1.9472 & 1.7741 & 1.7818 & $(1.3445,3.2655)$ \\
\hline & & & 0.1927 & 0.1776 & 0.1961 & 0.1939 & \\
\hline & 25 & $(2,2,1,0 * 22)$ & 2.0082 & 1.9694 & 1.8255 & 1.8320 & $(1.4059,3.1031)$ \\
\hline & & & 0.1597 & 0.1485 & 0.1572 & 0.1559 & \\
\hline \multirow[t]{4}{*}{40} & 30 & $\left(4,2,2,2,0^{*} 26\right)$ & 1.9999 & 1.9676 & 1.8456 & 1.8512 & $(1.4405,2.9642)$ \\
\hline & & & 0.1344 & 0.1269 & 0.1345 & 0.1335 & \\
\hline & 35 & $(3,0,1,1,0 * 31)$ & 2.0059 & 1.9780 & 1.8714 & 1.8763 & $(1.4777,2.8798)$ \\
\hline & & & 0.1204 & 0.1142 & 0.1183 & 0.1176 & \\
\hline
\end{tabular}

Table 5: Averaged values of MSEs for estimates of the reliability function, $P=0.6$

\begin{tabular}{cc|cccc}
\hline$n$ & $m$ & $\hat{S}_{M L E}$ & $\hat{S}_{S}$ & $\hat{S}_{L} c=0.1$ & $\hat{S}_{L} c=-0.1$ \\
& & $\mathrm{MSE}$ & $\mathrm{MSE}$ & $\mathrm{MSE}$ & $\mathrm{MSE}$ \\
\hline \multirow{2}{*}{5} & 5 & 0.92450 & 0.93668 & 0.92862 & 0.92834 \\
& & 0.001708 & 0.001433 & 0.001557 & 0.001559 \\
\cline { 2 - 6 } & 7 & 0.93224 & 0.94166 & 0.93648 & 0.93615 \\
& & 0.000932 & 0.000706 & 0.000830 & 0.000833 \\
\hline 20 & 10 & 0.93682 & 0.94376 & 0.94113 & 0.94077 \\
& & 0.000515 & 0.000370 & 0.000442 & 0.000446 \\
\cline { 2 - 6 } & 15 & 0.94021 & 0.94496 & 0.94457 & 0.94420 \\
& & 0.000316 & 0.000241 & 0.000268 & 0.000270 \\
\hline 30 & 20 & 0.94246 & 0.94606 & 0.94685 & 0.94647 \\
& & 0.000195 & 0.000154 & 0.000164 & 0.000165 \\
\cline { 2 - 6 } & 25 & 0.94402 & 0.94691 & 0.94844 & 0.94805 \\
& & 0.000139 & 0.000114 & 0.000121 & 0.000121 \\
\hline 40 & 30 & 0.94457 & 0.94699 & 0.94899 & 0.94860 \\
& & $1.173 \mathrm{e}-04$ & $9.869 \mathrm{e}-05$ & $1.033 \mathrm{e}-04$ & $1.028 \mathrm{e}-04$ \\
\cline { 2 - 6 } & 35 & 0.94526 & 0.94733 & 0.94969 & 0.94930 \\
& & $9.851 \mathrm{e}-05$ & $8.506 \mathrm{e}-05$ & $9.033 \mathrm{e}-05$ & $8.934 \mathrm{e}-05$ \\
\hline
\end{tabular}




\section{References}

[1] N. Balakrishnan, R. Aggarwala, Progressive Censoring: Theory, Methods, and Applications, Boston, MA: Birkhauser, 2000 .

[2] H.K. Yuen, S.K. Tse, "Parameters estimation for Weibull distributed lifetimes under progressive censoring with random removals" Journal of Statistical Computation and Simulation, 55, 1, (1996), 57-71.

[3] H.A. Zeinab, "Bayesian inference for the pareto lifetime model under progressive censoring with binomial removals", Journal of Applied Statistics, 35, 11, (2008), 1203-1217.

[4] S.J. Wu, C.T. Chang, "Inference in the Pareto distribution based on progressive censoring with random removals", Journal of Applied Statistics, 30, 2, (2003), 163-172.

[5] S.K. Tse and H.K. Yuen, "Expected Experiment Times for the Weibull Distribution under Progressive Censoring with Random Removals", Journal of Applied Statistics, 25, 1, (1998), 75-83.

[6] S.D. Grimshaw, "Computing maximum likelihood estimates for the generalized Pareto distribution",Technometrics $35,(1993), 185-191$.

[7] E. Castillo, A.S. Hadi, "Fitting the generalized Pareto distribution to data"' Journal of the American Statistical Association,92, (1997), 1609-1620.

[8] E. Castillo, A.S. Hadi,N. Balakrishnan,J.M. Sarabia, "Extreme Value and Related Models with Applications in Engineering and Science", Wiley, NewYork, 2005.

[9] C.C. Wu, S.F. Wu, H.Y. Chan, "MLE and the estimated expected test time for Pareto distribution under progressive censoring data", International Journal of Information and Management Sciences, 15, 3, (2004), 29-42.

[10] S.K. Tse,C. Yang,H. K. Yuen, "Statistical analysis of Weibull distributed lifetime data under Type II progressive censoring with binomial removals", Journal of Applied Statistics, 27, 8, (2000), 1033-1043.

[11] S.J. Wu, "Estimation for the two-parameter Pareto distribution under progressive censoring with uniform removals", Journal of Statistical Computation and Simulation, 73 (2003), 125134.

[12] W. Shuo-Jye and C. Chun-Tao, "Inference in the Pareto Distribution Based on Progressive Type II Censoring with Random Removals", Journal of Applied Statistics, 30, 2, (2003), 163-172.

[13] A. Basu, N. Ebrahimi, "Bayesian approach to life testing and reliability estimation using asymmetric loss function", Journal of Statistical Planning and Inference 29, (1991), 21-31. 